\title{
Active Control of Solar Array Dynamics During Spacecraft Maneuvers
}

\author{
Brant A. Ross ${ }^{1}$ and Nelson $\mathrm{Woo}^{2}$ \\ MotionPort LLC, St George, UT 84790 \\ Thomas G. Kraft ${ }^{3}$ \\ NASA Glenn Research Center, Cleveland, OH 44135 \\ and \\ Joseph R. Blandino ${ }^{4}$ \\ Virginia Military Institute, Lexington, VA 24450
}

\begin{abstract}
Recent NASA mission plans require spacecraft to undergo potentially significant maneuvers (or dynamic loading events) with large solar arrays deployed. Therefore there is an increased need to understand and possibly control the nonlinear dynamics in the spacecraft system during such maneuvers. The development of a nonlinear controller is described. The utility of using a nonlinear controller to reduce forces and motion in a solar array wing during a loading event is demonstrated. The result is dramatic reductions in system forces and motion during a 10 second loading event. A motion curve derived from the simulation with the closed loop controller is used to obtain similar benefits with a simpler motion control approach.
\end{abstract}

\section{Introduction}

$\mathrm{T}$ YPICALLY it has been sufficient to consider the dynamics of a large deployable space structure only during deployment. Once the space structure was deployed it has been reasonable to expect that subsequent spacecraft maneuvers would be gradual given the assumption of an orbiting spacecraft. More recently NASA mission plans have included spacecraft that travel in inter-planetary space with large solar arrays deployed. For example it has become necessary to assess the dynamic response of the motion and loads of a large solar array in response to a reference $0.1 \mathrm{~g}$ acceleration applied normal to the plane of the array (a loading event).

NASA $^{1}$ has stated that certain mission scenarios for spacecraft with large (300 kW class) solar arrays could include chemical thrust loads as well as transient docking loads and RCS plume loads. Preliminary analysis has shown that design loads for solar arrays may reach an acceleration of $0.2 \mathrm{~g}$ (includes a dynamic amplification factor of two). The preliminary design stiffness assumptions for these solar arrays would correspond to a minimum natural frequency of $0.1 \mathrm{~Hz}$, which is anticipated to be difficult to achieve as larger solar arrays are developed.

The simulation of large flexible space structures has often been done as a linear dynamics problem, considering a combination of modal representations of the flexible bodies. But as solar arrays increase in size nonlinear dynamics techniques are increasingly needed in order to consider large displacements in the structures as well as the momentum of the sections of the structure in motion. This nonlinear simulation of large structures can be challenging, however, as including the large motions in large space structures such as solar arrays in simulations can cause nonlinear Finite Element Analysis (FEA) software to have convergence problems and the run times can be long. Therefore existing nonlinear FEA software may not be the best method to simulate structural assemblies with significant motion.

\footnotetext{
${ }^{1}$ Business Manager, 473 S. River Road.

${ }^{2}$ Application Engineer, 473 S. River Road.

${ }^{3}$ Mechanical Engineer, 21000 Brookpark Road.

${ }^{4}$ Professor, Dept. of Mechanical Engineering, and AIAA Associate Fellow
} 
A promising alternative is to use Multi-Body Dynamics (MBD) software that can model nonlinear flexible bodies and includes solution techniques that efficiently handle large motions in a mechanical assembly. An acceptable MBD software ${ }^{2-7}$ was identified for this application that can simulate nonlinear (in deformation) meshbased flexible bodies. It also has the ability to "extract" a model, or use the end conditions of one simulation as the starting point for another simulation. The new model retains the strain information of the flexible structure corresponding to the loaded condition of the previous step such that the flexible structure is correctly pre-stressed.

The MBD software, RecurDyn, was used to simulate the packaging and deployment of a slit-tube solar array wing. Details of this simulation are presented in Ref [8]. The operations that were modeled included forming the slit tube boom to the mandrel, rolling the tube onto the mandrel, and then deploying the tube from the mandrel. A critical step was forming the tube to the mandrel as this involves flatting the tube, a process that involves introducing significant strain energy into the tube. This is important because during deployment the strain energy is released as the flattened tube returns to its semi-circular shape. Figure 1 shows five of the steps in the process of applying a pressure to the end of a generic slit tube in order to cause it to form around the mandrel. These steps a-e illustrate a pressure being applied to portions of the tube to press it up against the mandrel. As a result the tube flattens and stress develops within the tube. The pressure continues until the flattened tube is formed to the shape of the mandrel. A rigid element, much like a Nastran RBE2 element is added to fix the end of the tube to the mandrel and the tube is then ready to be rolled into a stowed configuration.

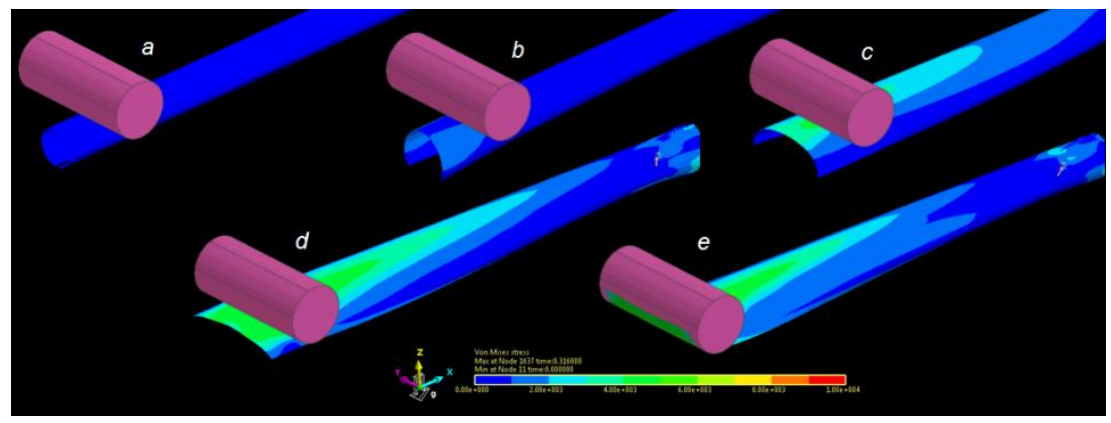

Figure 1. Simulation of the Forming Process of a Slit Side Tube

The slit tube solar array wing consists of two slit tubes, a solar blanket, a frame body, and a mandrel, as shown in Figure 2. The frame and mandrel are modeled as rigid bodies. The frame is assumed to be attached to the spacecraft and supports one end of the tubes and the solar blanket. The tubes and blanket are rolled around the mandrel when the solar array wing is stowed. The tubes and solar blanket are modeled with finite element meshes comprised of shell elements. The tubes are typically made using a composite material. In this study the average orthotropic properties in the axial and transverse direction are used in the tube meshes. The solar blanket generally consists of a flexible woven mesh with the solar cells attached. The primary effect of the solar blanket on the dynamic behavior of the solar array wing is due to its mass, and the stiffness has little effect. The element and material properties of the solar blanket mesh were set to correctly represent the distributed mass of the blanket and low stiffness.

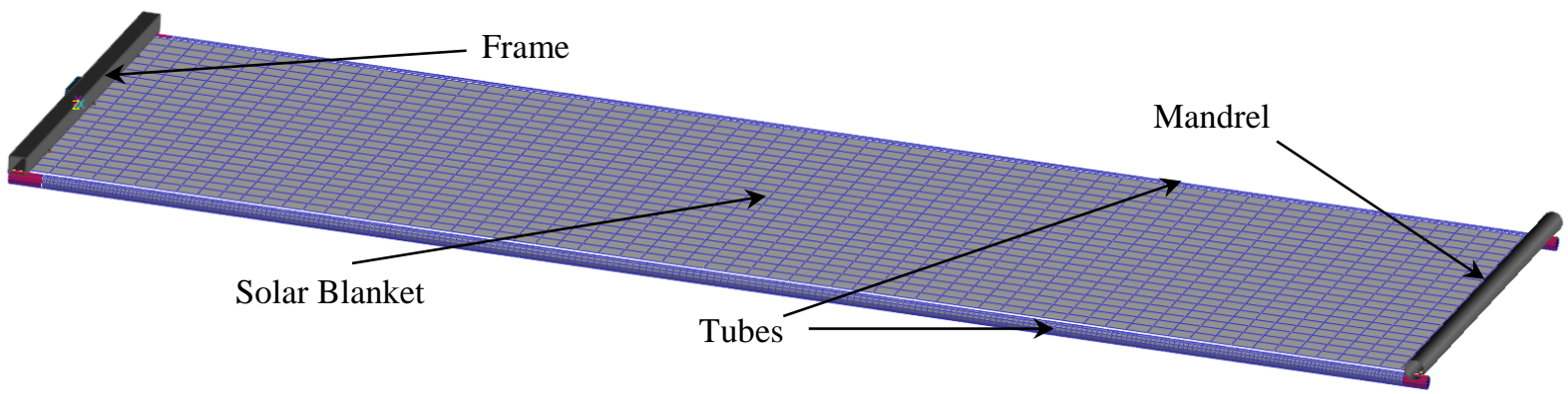

Figure 2. The Slit Tube Solar Array Assembly

With the boom in the deployed configuration an MBD simulation was done of the solar array as it underwent a $0.1 \mathrm{~g}$ acceleration for 10 seconds (chemical thrust event). The simulation revealed a substantial bouncing motion due to the combination of the mass contribution of the solar blanket, the substantial length of the array, and the modest stiffness of the tubes. The deflection of the tubes and the relative motion of the mandrel (and hence the solar 
blanket) were large enough for the momentum of the distributed mass in the solar blanket to cause the bouncing dynamics. The attachment loads of the solar array wing on the frame increased substantially as the structure reached the extremes of each vibrational cycle, corresponding to higher loads (and stresses) in the supporting slit tubes.

Mass constraints related to mission requirements may discourage the use of additional material (increased thickness of the tubes) to stiffen the structure in order to reduce the magnitude of the bouncing motion. Therefore it may be necessary to use active control in the design of future large deployable solar arrays to meet critical power per deployed area, power per packaged volume and basic frequency requirements.

The active controller could reduce vibration-induced loading in the solar array structure to reduce overall loads and cause the structure to act as if it were stiffer. A MBD model of the solar array wing could include a controller at the gimbal in order to provide active control to reduce the bouncing effects that can occur during spacecraft transient dynamic loading events. MBD software includes modules for controller definition and a robust interface between the controller and the mechanical model. Simulation could not only efficiently and accurately assess the effectiveness of the controller but also the control structure interaction between the large flexible solar array wing structures, the local controllers, and the spacecraft attitude control system.

\section{Model Characterization}

The first step in this study was to create a generic roll-up tube solar array wing model as depicted earlier in Figure 2. The slit tubes were meshed specifically for simulating the deployed solar array wing, with elements that are longer in the axial direction than they would otherwise be created for packing and deployment simulations where the tube material undergoes extreme bending. The resulting full mechanical model of the solar array wing has a high fidelity and can be simulated quickly. A simulation of a 10 second thrust load event can be done in 5-7 minutes on a standard desktop computer. Figure 3 shows the solar array wing at the maximum deformation that occurs during the simulation of a steady $0.1 \mathrm{~g}$ thrust load event.

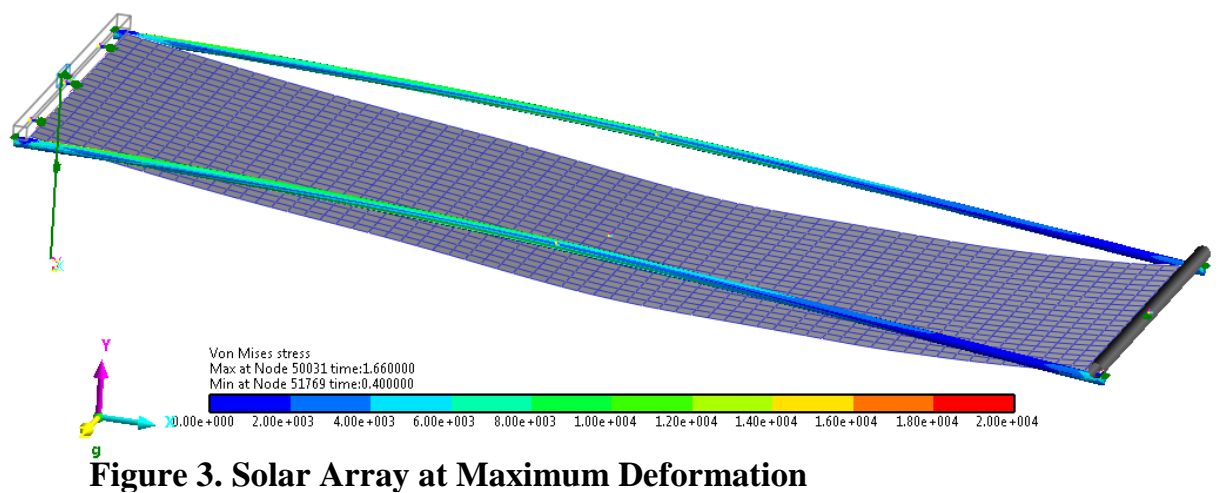

The next task was to characterize the dynamic response of the solar array wing structure. It is assumed that the controller can only influence the rotational degree-of-freedom of the solar array wing frame with respect to the spacecraft. The controller provides a signal to an actuator which imposes a rotational torque on the mount between the spacecraft and solar array wing frame. It is significant to understand the relationship between the flexing of the tubes of the solar array wing and the corresponding torques at the frame mount so that the controller can be designed properly.

A simulation of the solar array wing was done under an acceleration of $0.1 \mathrm{~g}$. The results, as shown in Figure 4, show a good correlation between the vertical $(+Y)$ displacement between the mandrel and the frame to the resulting torque where the frame attaches to the spacecraft. This vertical displacement is also referred to as "windup" in this paper. Since the frame can rotate, the vertical displacement must be measured relative to the moving coordinate system of the frame, as shown in the Figure 5.

A practical closed loop controller design should consider how the information that the controller needs can be gathered from the solar array wing structure. Displacement and velocity information of the mandrel with respect to the solar array base frame are needed. However it is not easy to gather such information.

Therefore the instrumentation concept is to measure the relative acceleration between the mandrel and the frame and integrate the relative acceleration in order to obtain the needed information. This approach can be implemented simply by mounting accelerometers within the mandrel and was mocked-up in the simulation model. The plot in Figure 6 shows how a slight offset between the measured points on the mandrel and frame remains constant during 
the simulation. One curve is the model-predicted displacement and the other curve is the displacement derived from the acceleration data.

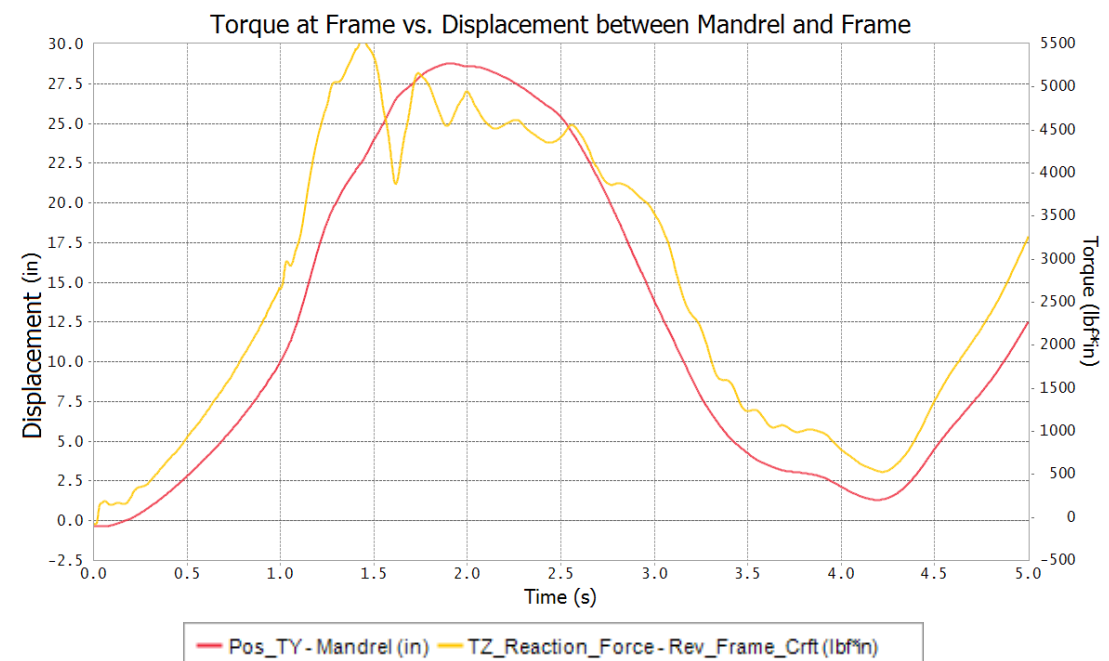

Figure 4. Correlation Between Tube Tip Displacement (Windup) and Torque at the Frame

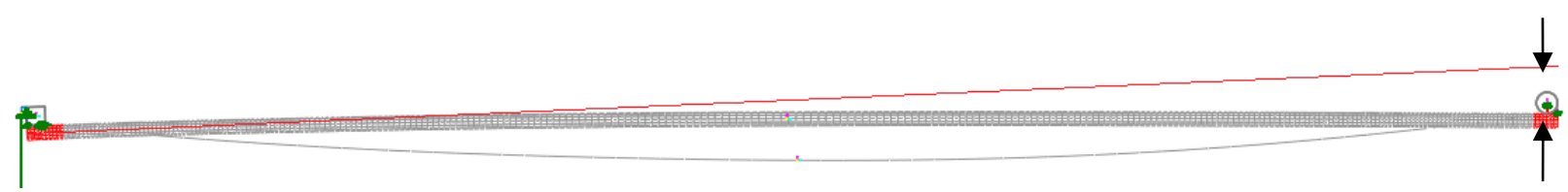

Figure 5. Determination of Tube Tip Displacement With Respect to the Frame

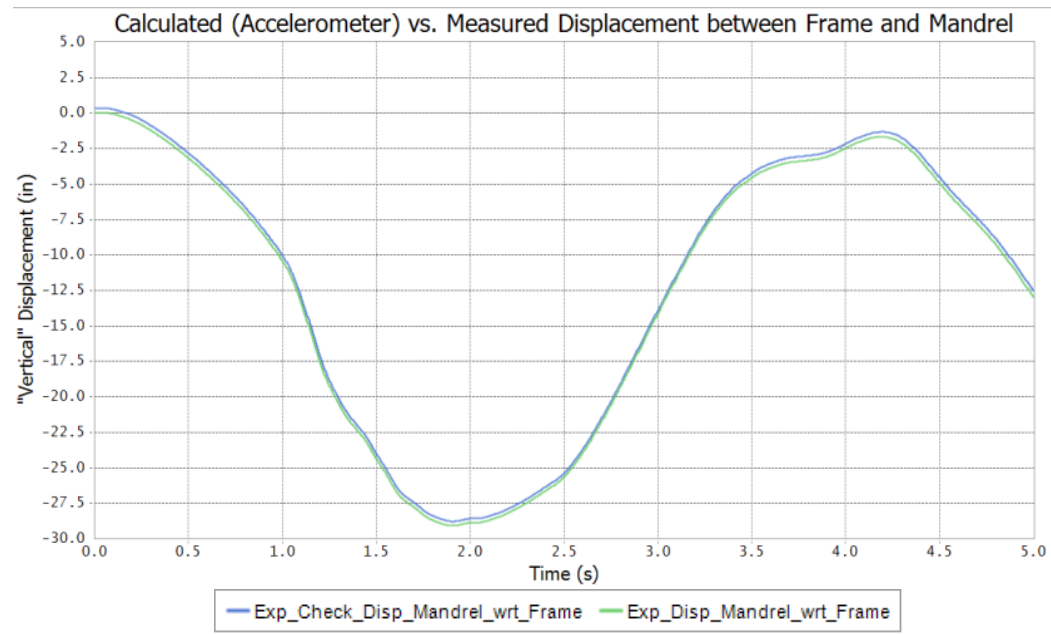

Figure 6. Comparison of Pseudo-Measured Displacement vs Actual Displacement

An error term of 3.5\% (based upon accelerometer specifications and allowable mounting error) is introduced in the model in order to check the robustness of the controller when the accelerometers are not functioning perfectly or mounted in a position in the mandrel that is not locally vertical (aligned with the $+Y$ axis with respect to the frame) when the solar array wing is deployed. The final position of the mandrel after deployment is not certain and a perfectly mounted accelerometer (before rollup and deployment) could develop an alignment error if the solar array wing does not roll out completely and the mandrel is not returned to its original position.

The model was parameterized in order to facilitate rotating the solar array wing at different speeds during the acceleration event in order to determine the response of the system to rotational velocity. The resulting rotational 
velocities of the solar array wing during the various simulations are shown in the plot of frame A of Figure 7. The windup in the solar array wing for the cases of different rotational velocities is shown in frame B of Figure 7.
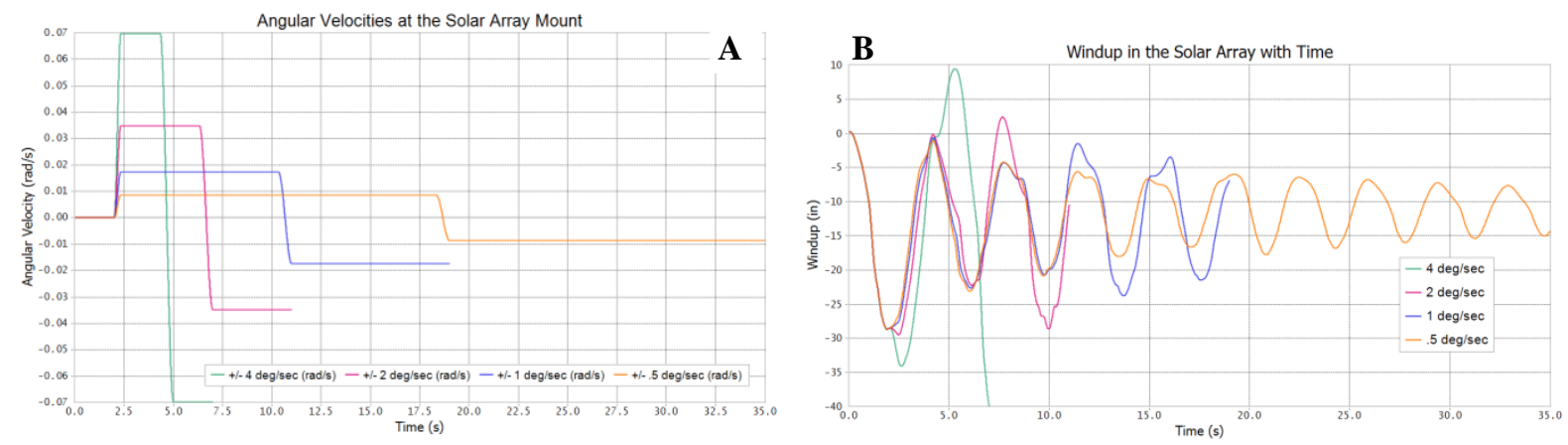

Figure 7. Design Study Input Velocities and Corresponding Results

An interesting result of the design study is the relationship between the torque at the frame mount and the windup in the solar array wing is consistent within a certain range of windup, as shown in Figure 8. Note that if the windup is too high then the torque values diverge for the various velocities. The resulting line has a slope of -243.3 in-lbf per inch and shows 194.9 in-lbfs of torque at a zero windup during the $0.1 \mathrm{~g}$ thrust event. The fact that the torque does not significantly depend on velocity is a confirmation that there is little structural damping in the solar array wing structure, given a damping value of $0.1 \%$ for the materials used to define the tubes and solar blanket.

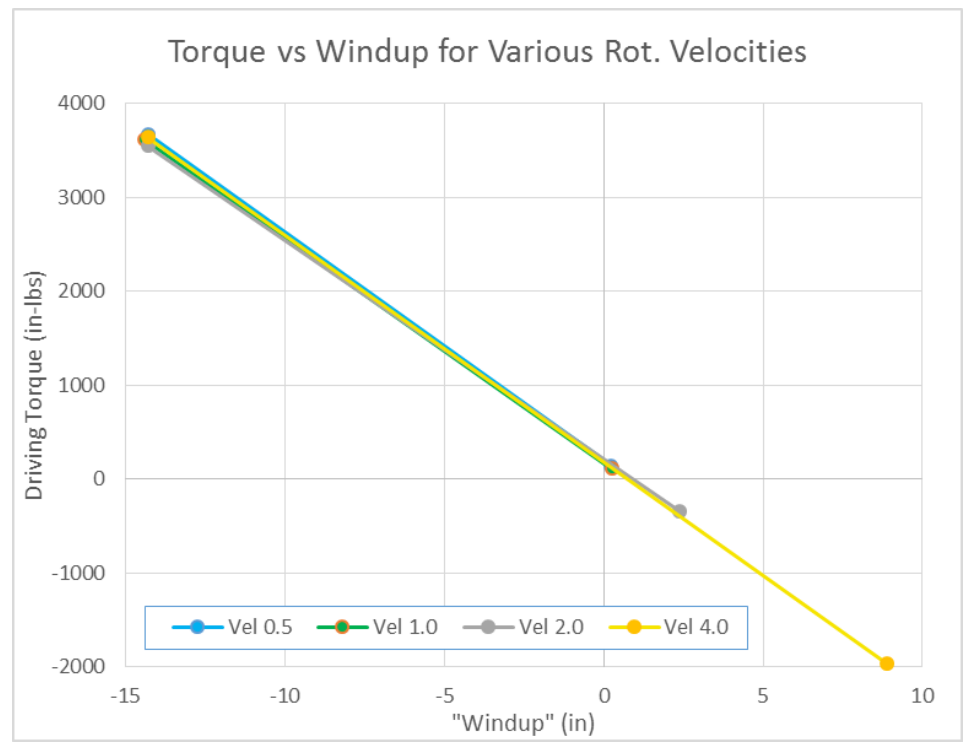

Figure 8. Torque vs Windup for Various Rotational Velocities

The solar array wing has a deformed shape in dynamic equilibrium that is related to the $0.1 \mathrm{~g}$ acceleration. In the passive configuration, where the frame is fixed to the spacecraft, the solar array wing deforms to assume the equilibrium shape and then overshoots that configuration. The desired behavior would be to deform to the equilibrium shape with minimum motion. A review of the animations suggested that the best possible situation would be for the controller to rotate up the frame such that the deformed shape would be oriented to be as close as possible to the position of the unloaded solar array wing. Under acceleration the solar blanket will sag and this can't be controlled. Therefore the rotation of the fame should raise the tubes while the blanket sags, as shown in Figure 9. An inspection of the animation frames from the simulation results of the design study suggested that the optimal rotational angle is 2.25 degrees. 


\section{Control System Development}

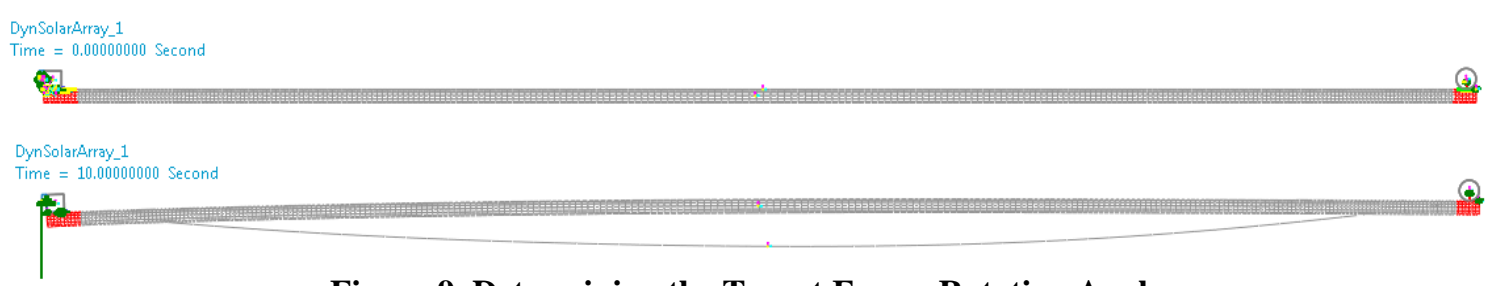

Figure 9. Determining the Target Frame Rotation Angle

The guiding parameters for the controller design, shown in Table 1, were determined by examining the results of the characterization simulation runs as well as the design study runs. The nominal torque and windup occur at the dynamic equilibrium point for the $0.1 \mathrm{~g}$ thrust event. A controller was defined using the RecurDyn control simulation module, CoLink, as shown in the block diagram in Figure 10. The RecurDyn block represents the entire mechanical assembly of the solar panel. The input to the RecurDyn block is the torque that is being applied at the mount. The outputs of the RecurDyn block are from the virtual sensors that are used by the controller, namely: 1) the windup of the solar array wing, 2) the angle of the frame to the spacecraft, and 3) the rotational velocity between the frame and the spacecraft. Note that the rotational velocity term is not used in the controller but is available in case the derivative information would be helpful with a future version of the controller.

The relationship between the torque and the windup is used to limit the torque according to the current windup of the solar array wing. The precise windup that is output from the mechanical model is scaled by a factor of 0.965 to reflect the $3.5 \%$ error mentioned previously.

The target of the controller is to achieve the previously-determined optimal rotation of 2.25 degrees while not causing an excessive windup. Two Min blocks are used to prevent excessive torque. The leftmost Min block outputs the minimum of the requested torque due to the angular error and the available torque that can be applied given the existing windup. The rightmost Min block ensures that the torque does not exceed the specified maximum torque.

Table 1. Target Controller Values

\begin{tabular}{|l|l|}
\hline Model Output & Target \\
\hline Maximum Torque & 7000 in-lbf \\
\hline Nominal Torque & 2905 in-lbf \\
\hline Maximum Speed & $4 \mathrm{deg} / \mathrm{sec}$ \\
\hline Maximum Windup & 35 inches \\
\hline Nominal Windup & 11.25 inches \\
\hline Target Rotation & 2.25 degrees \\
\hline
\end{tabular}

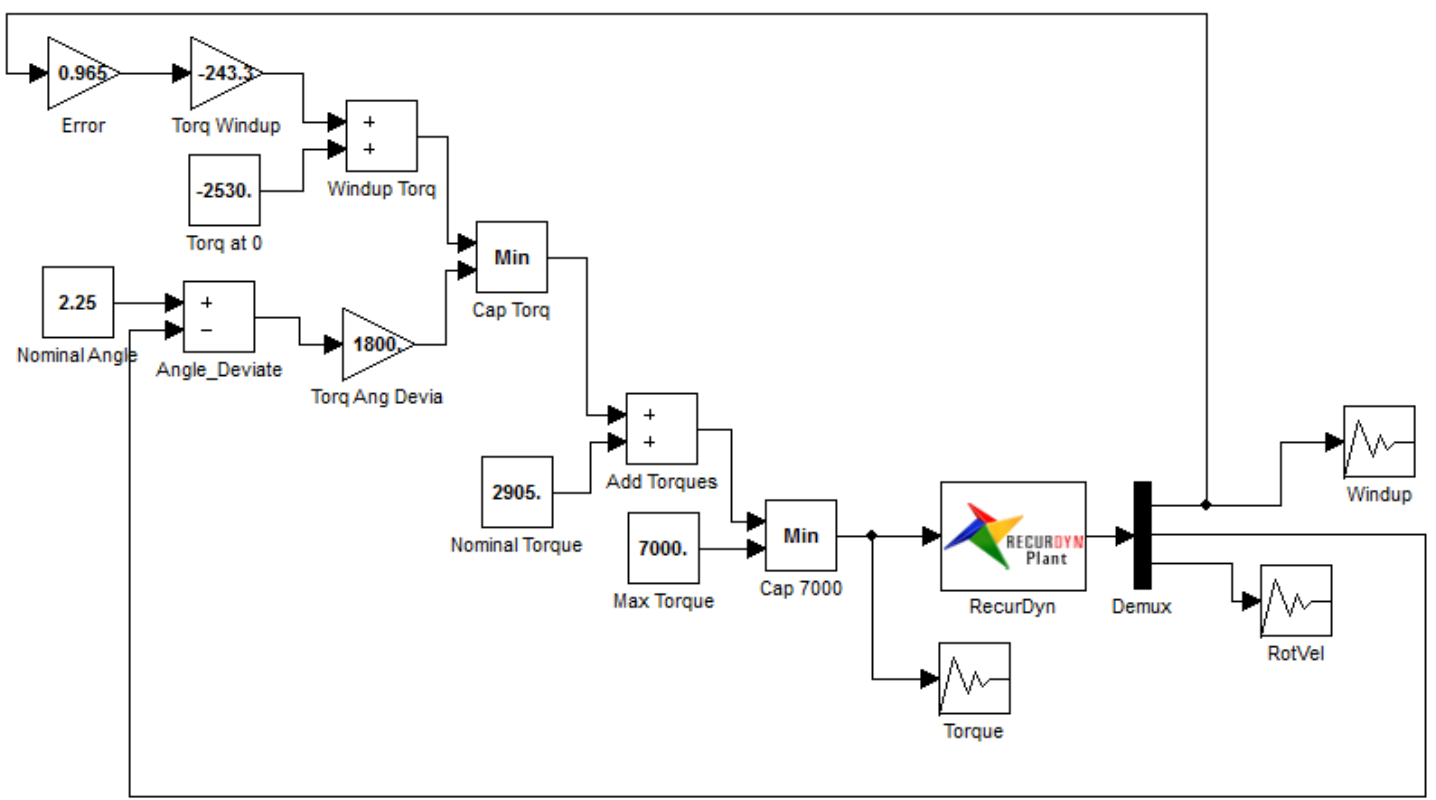

Figure 10. Non-Linear Controller Design

\section{Results}

Results from the first set of simulations show that the controller worked very well, significantly reducing the motion and torque at the base of the solar array wing during 10 seconds of the thrust event. Figure 11 compares the

6

American Institute of Aeronautics and Astronautics 
motion of the mandrel with respect to the frame for the baseline (fixed frame) configuration and the dynamically controlled cases. The maximum relative motion (windup) of the mandrel with the baseline configuration is approximately 28 inches.

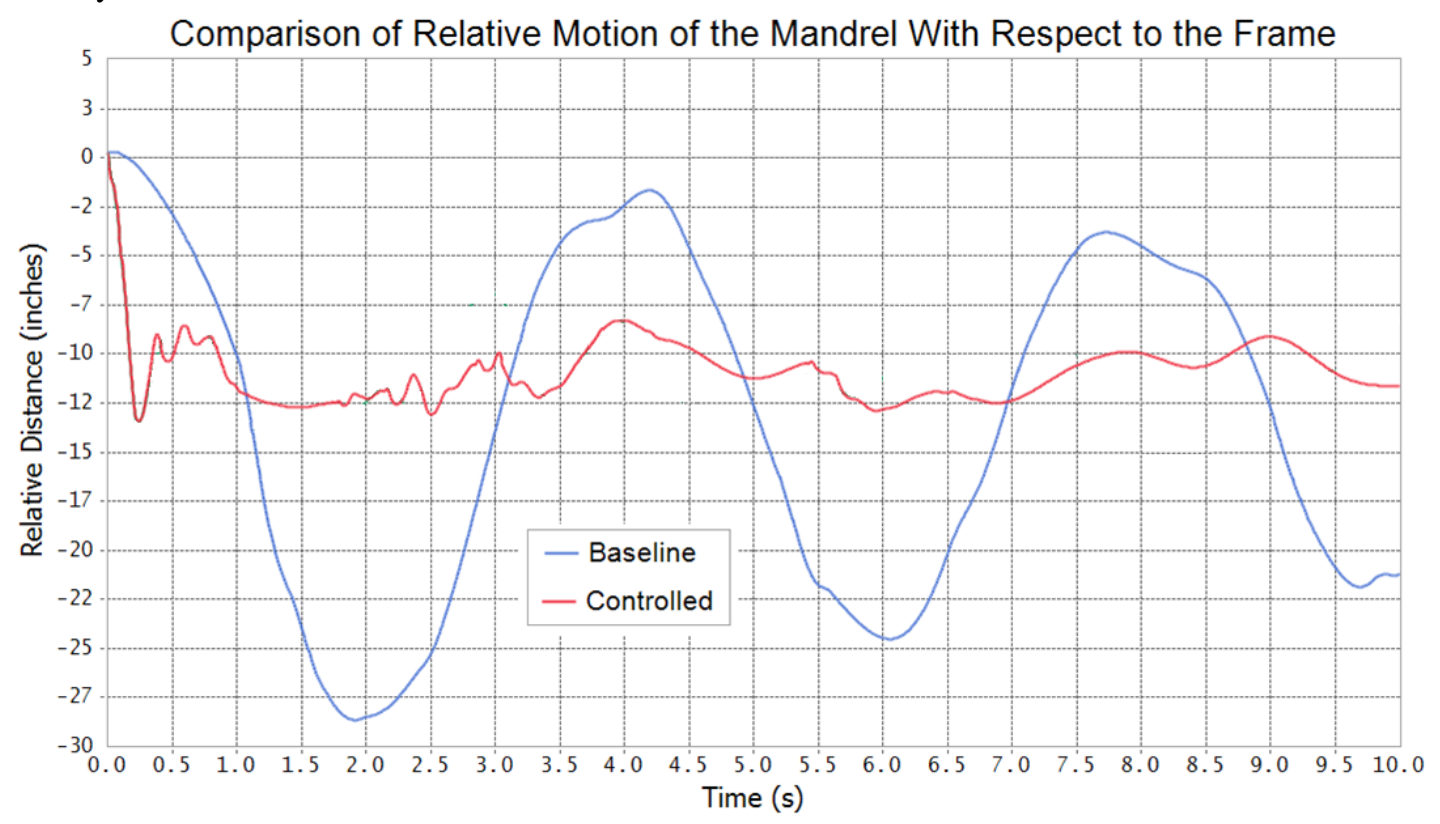

Figure 11. Windup Comparison

The maximum relative motion (windup) of the mandrel with the actively controlled configuration is approximately 13 inches. Given that the nominal (equilibrium) relative motion of the mandrel in a $0.1 \mathrm{~g}$ event is 11.25 inches, this is almost a $90 \%$ reduction of the motion beyond the dynamic equilibrium configuration.

There is also a benefit in the vibrational response of the structure. In the baseline case the peak-to-peak time duration is approximately 4 seconds, or approximately a $0.25 \mathrm{~Hz}$ vibrational response. While this frequency is larger than the target of $0.1 \mathrm{~Hz}$ for the array, it should be considered that a total solar array assembly would consist of pairs of solar panels such as this example model that are extended out for a number of segments. Therefore it may be necessary for each panel to have a primary frequency of $1.0 \mathrm{~Hz}$ or more.

In the actively controlled case there is no consistent vibration behavior, with distances between peaks from $1-$ 1.5 seconds. Therefore the response of the dynamically controlled case has an apparent primary frequency that is much closer to the $1.0 \mathrm{~Hz}$ frequency that is often desired with larger space structures. Further improvements to the controller could result in an apparent frequency response that is even more favorable. This improved behavior reduces the demands on the spacecraft attitude control system.

Figure 12 compares the torque at the frame for the baseline (fixed frame) configuration and the dynamically controlled cases. The maximum torque at the frame with the baseline configuration is approximately $5530 \mathrm{in}-\mathrm{lbf}$. The maximum torque at the frame with the actively controlled configuration is approximately 3470 inches. Given that the nominal (equilibrium) torque at the frame in a $0.1 \mathrm{~g}$ event is $2625 \mathrm{in}-\mathrm{lbf}$, this is almost a $79 \%$ reduction of the torque beyond the dynamic equilibrium configuration.

\section{Refined Approach}

For a given mission there could be a reasonably limited number of thrust events (varied thrust values and durations) such that simulations could be done in advance to predetermine a set of desired rotational profiles for the solar array wing frame. It is hoped that such an approach could provide the substantial advantage of not requiring any sensors and no closed loop control algorithm is needed. Instead it is a simply a matter of controlling the displacement of a stepper motor based upon the calculated motion of the fully controlled model.

The feasibility of such an approach was evaluated for this model. The rotational motion of the frame using the controller is shown in the frame A of Figure 13 and a derived smooth motion function is shown in frame B. The smoothed velocity profile data was then used in a new model to directly control the frame rotation at the base of the solar array wing. 


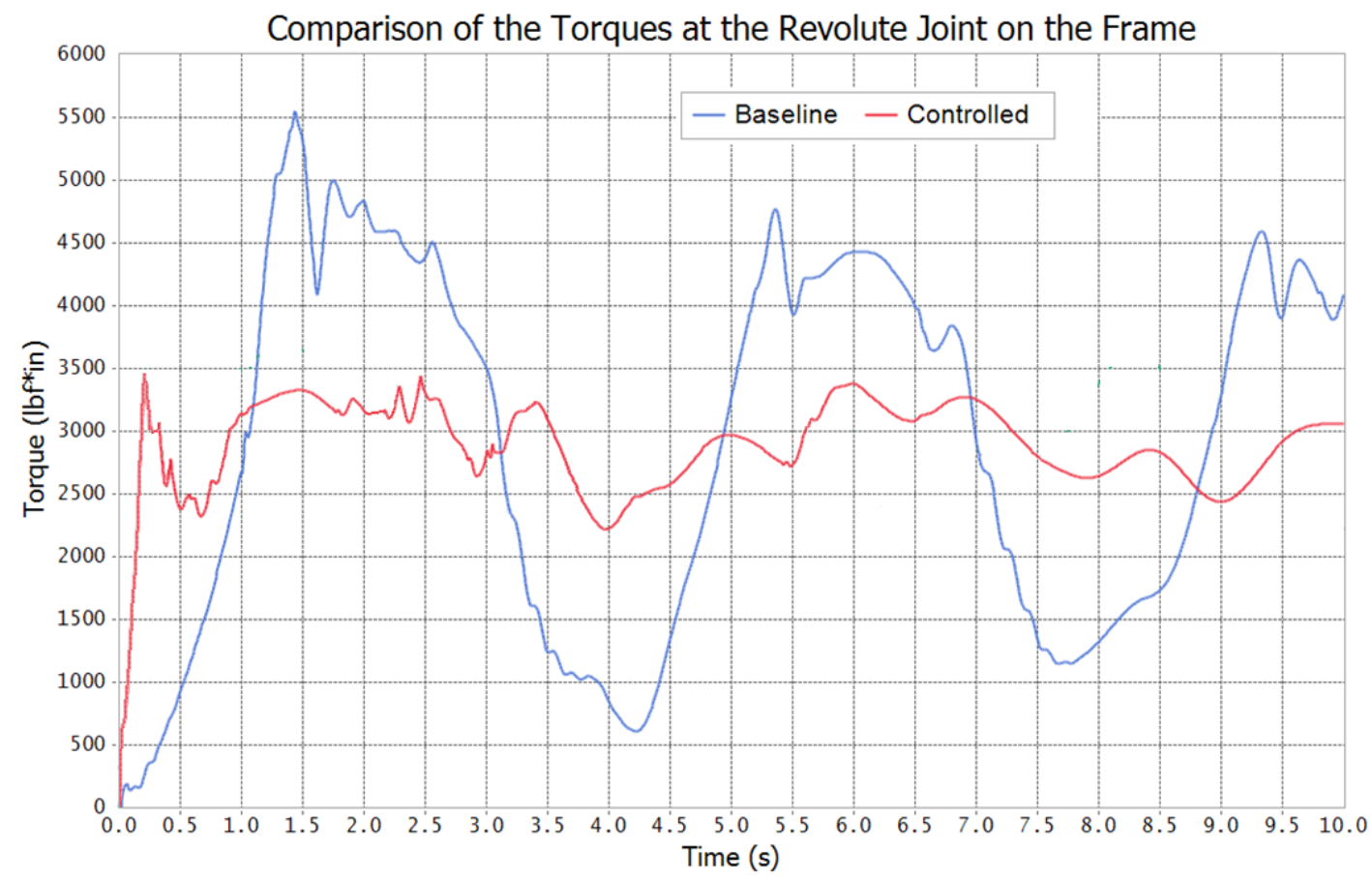

Figure 12. Reaction Torque Comparison
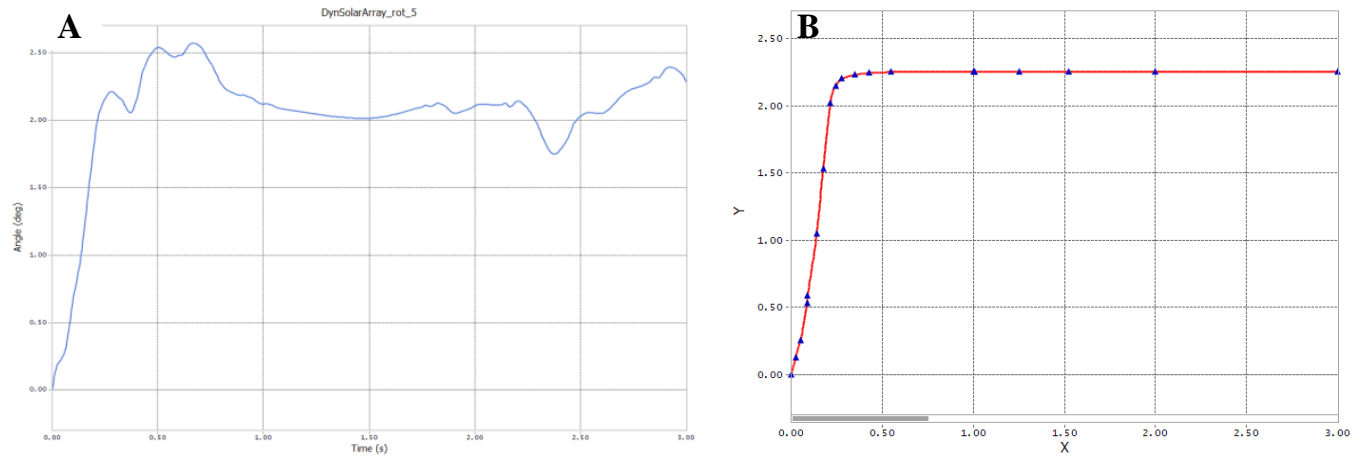

Figure 13. Derived and Smooth Base Rotation

Figure 14 compares the motion of the mandrel with respect to the frame for the baseline (fixed frame) configuration, the dynamically controlled case and the predefined motion case. It can be seen that the use of a predefined motion results in much of the benefit of using a closed loop controller. The maximum relative motion of the mandrel with the predefined motion increases to approximately 14 inches, or more than an $83 \%$ reduction of the motion beyond the dynamic equilibrium configuration.

Figure 15 compares the torque at the frame for all three cases. The maximum torque at the frame with the predefined motion is approximately 3700 in-lbf, for almost a $70 \%$ reduction of the torque beyond the dynamic equilibrium configuration as compared to the baseline case. Again the predefined motion case shows much of the benefit of using a closed loop controller.

With the predefined motion case the improved vibrational response continues to be observed, with the longest peak-to-peak interval being less than 1.5 seconds. Just like with the closed loop controller, further improvements to the motion profile can result in an even better frequency response. 


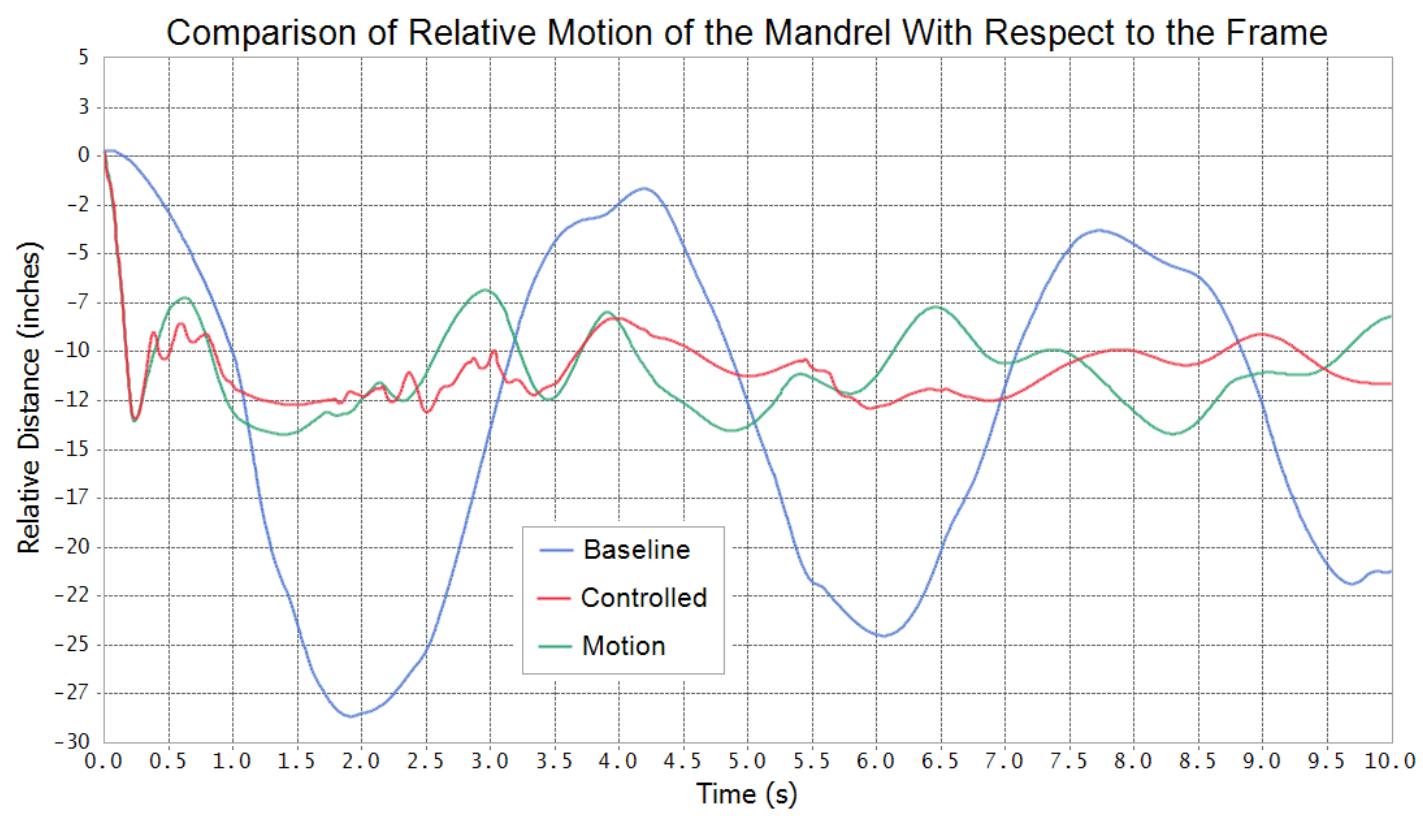

Figure 14. Windup Comparison Including Predefined Motion

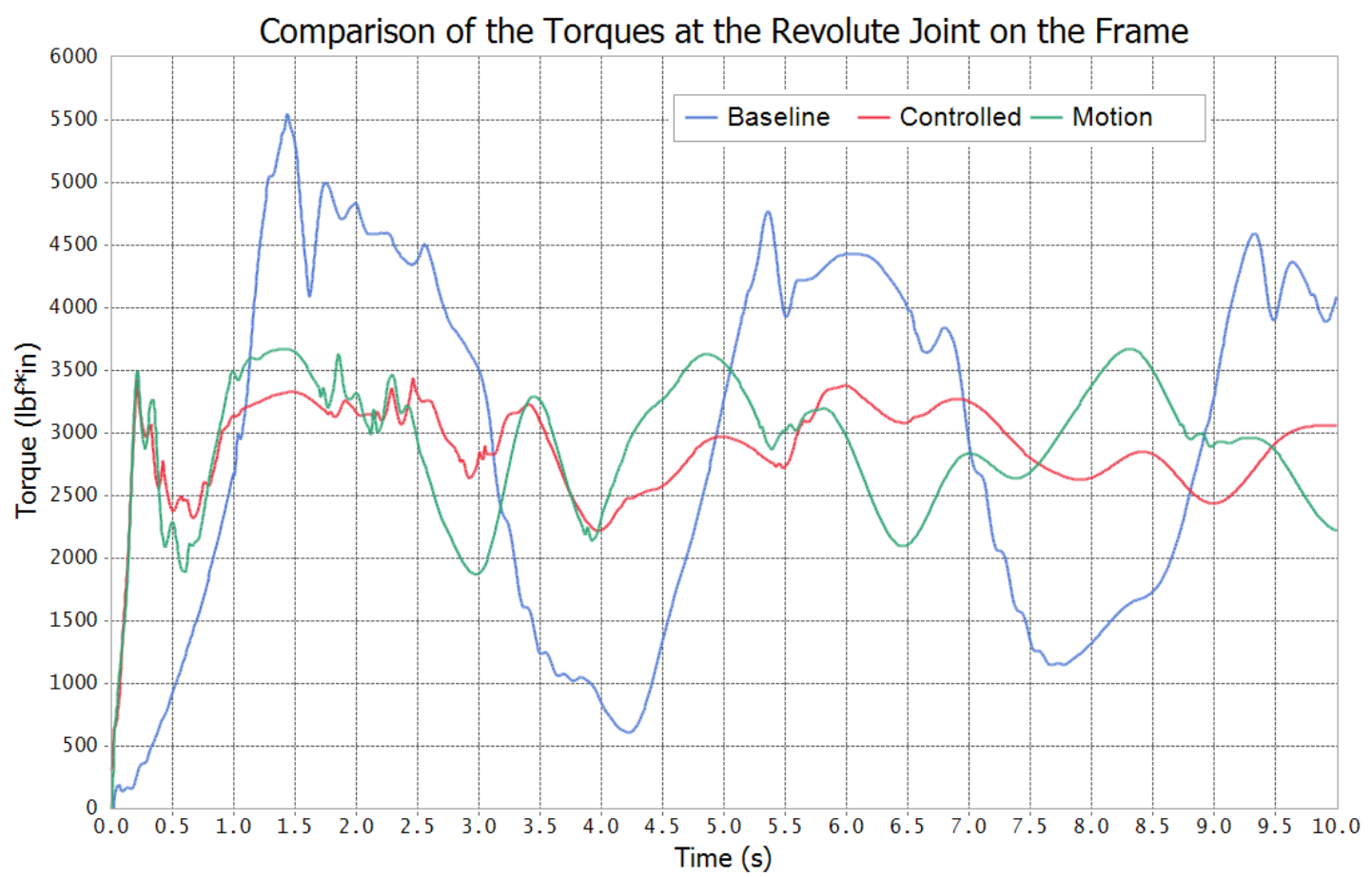

Figure 15. Reaction Torque Comparison Including Predefined Motion

\section{Conclusions}

Given the interest in developing spacecraft with missions that include various transient loading events while large flexible structures such as solar arrays are deployed, there is a need to validate the dynamic behavior of the deployed structures. MBD software with the ability to model nonlinear flexible bodies is well suited to simulate such dynamics, including momentum effects. The dynamics cases were modeled and simulated; fixing the frame to the spacecraft, using a closed loop controller to apply torque to a frame pivot, and pivoting the frame according to a predefined motion. Simulation results clearly indicate that the dynamic behavior of the rollup solar array wing with 
nonlinear control and predetermined motion of the frame is better and safer than statically/rigidly mounting the frame of the solar array wing. Additional application of this approach should be done with various other solar array wing configurations and different solar array wing sizes as well as with other large/flexible deployable space structures.

\section{Acknowledgments}

B. A. Ross and the other authors thank NASA for support of this research under SBIR Contract NNX14CC72P, "Simulating Nonlinear Dynamics of Deployable Space Structures."

\section{References}

${ }^{1}$ David J. Hoffman, et. al., "Concept Design of High Power Solar Electric Propulsion Vehicles for Human Exploration", NASA/TM-2011-217281, IAC-11-D2.3.5, http://ntrs.nasa.gov/archive/nasa/casi.ntrs.nasa.gov/20120000068_2011025608.pdf, December 2011.

${ }^{2}$ RecurDyn V8R4 Help Documenation, FunctionBay, Inc., Seoul, Republic of Korea, July 2015.

${ }^{3}$ H. J. Cho, et. al. "A Recursive Implementation Method with Implicit Integrator for Multibody Dynamics," Proceedings of ASME Design Engineering Technology Conference, Third Symposium on Multibody Dynamics and Vibration, September 9-12, 2001, Pittsburgh, PA, USA.

${ }^{4}$ Dae Sung Bae, "Development of a New Multi-Flexible Body Dynamics (MFBD) Platform: A Relative Nodal Dis-placement Method for Finite Element Analysis," Proceedings of the ASME 2005 IDETC \& CIE Conference, September 24-28, 2005, Long Beach, California USA, DETC2005-84723.

${ }^{5}$ Brant A. Ross, "History, Perspective and Outlook for Media Transport Simulation Using Multibody Dynamics," Proceedings of the ASME IDETC 2005 Conference, ASME Fifth Symposium on Multibody Dynamics and Vibration, September 24-28, 2005, Long Beach, CA, DETC2005-85191.

${ }^{6}$ Brant A. Ross and Nelson Woo, "Early Experiences in Using Multibody Dynamics Software to Simulate Flexible Webs in Production," Proceedings of the ASME IDETC 2009 Conference, ASME Seventh Symposium on Multibody Dynamics and Vibration, August 30 - September 2, 2009, San Diego, California, USA, DETC2009-86719.

${ }^{7}$ Juhwan Choi, Jin Hwan Choi, A Smooth Contact Algorithm Using Cubic Spline Surface Interpolation for Rigid and Flexible Bodies, Proceedings of the ASME IDETC 2013 Conference, ASME Ninth Symposium on Multibody Dynamics and Vibration, August 4-7, 2013, Portland, Oregon, USA, DETC2013-12117.

${ }^{8}$ Ross, B.A., Woo, N., Blandino, J.R., "Simulating Non-Linear Deployment Dynamics," The 2 ${ }^{\text {nd }}$ AIAA Spacecraft Structures Conference, 2-8 January, 2016, San Diego, California, USA. 